ICRR-Report-519-2005-2

December 8, 2018

\title{
Hadronic EDM Constraints on Orbifold GUTs
}

\author{
Junji Hisano, Mitsuru Kakizaki and Minoru Nagai \\ ICRR, University of Tokyo, Kashiwa 277-8582, Japan
}

\begin{abstract}
We point out that the null results of the hadronic electric dipole moment (EDM) searches constrain orbifold grand unified theories (GUTs), where the GUT symmetry and supersymmetry (SUSY) are both broken by boundary conditions in extra dimensions and it leads to rich fermion and sfermion flavor structures. A marginal chromoelectric dipole moment (CEDM) of the up quark is induced by the misalignment between the $\mathrm{CP}$ violating left- and right-handed up-type squark mixings, in contrast to the conventional four-dimensional SUSY GUTs. The up quark CEDM constraint is found to be as strong as those from charged lepton flavor violation (LFV) searches. The interplay between future EDM and LFV experiments will probe the structures of the GUTs and the SUSY breaking mediation mechanism.
\end{abstract}


After the discovery of successful agreement of the three extrapolated gauge couplings at a higher energy scale, supersymmetric grand unified theories (SUSY GUTs) have been considered as ones of the most predominant candidates for theories beyond the standard model (SM). The ratio of the bottom quark to tau lepton masses supports the idea of GUTs which also predict the Yukawa coupling unification. There have been extensive researches to detect further implications for SUSY GUTs.

Crucial hints for SUSY GUTs are also obtained in flavor and CP violation originated from the SUSY breaking scalar mass terms. Because SUSY GUTs also provide some relations between squark and slepton mass matrices [1,2], we can probe structure of SUSY GUTs by combining results of low energy experiments, such as $K, B$ physics, lepton flavor violating (LFV) decays and electric dipole moment (EDM) searches.

Recently it was pointed out that null results of hadronic EDM experiments severely constrain CP-violating squark flavor mixings in SUSY models [3, 4]. When off-diagonal elements in squark mass matrices have sizable imaginary parts, unacceptably large chromoelectric dipole moments (CEDMs) would be induced and conflict with hadronic EDM data. The flavor conserving moments, which are induced by loop diagrams, depend on the flavor violating mass parameters via the internal lines, and they are enhanced by heavier quark masses due to a chirality flip nature of the dipole moment operators. In particular the bound on down-type squark mixings between the second and third generations plays a crucial role in discussing the SUSY contribution to the CP asymmetry in the $B$ meson system. If a sizable deviation from the SM prediction of the CP asymmetry in $B_{d}^{0} \rightarrow \phi K_{S}$ [5] is attributed to the right-handed down-type squark mixing, which is radiatively generated by the right-handed neutrino Yukawa coupling in SUSY GUTs [6], the CEDM of the strange quark would exceed the experimental bound. We emphasize that the hadronic EDM constraints are important criteria for distinguishing various scenarios and for building models [3, 4, 7, 8, 9, 10].

In constructing realistic SUSY GUT models, we are confronted with many difficulties. Baryon number violating dimension five operators induced by color triplet Higgs multiplet exchange have to be suppressed in order to stabilize proton adequately [11]. The mass ratios of the down-type quarks to charged leptons in the first two generations are quite different from the counterpart of the third generation. In addition, a vast mass hierarchy between the light Higgs doublets and their heavy color triplet partners is required without invoking a fine-tuning of parameters.

There are many attempts to solve the problems inherent in SUSY GUTs. Approaches employing extended representations and/or groups are well investigated [12]. Meanwhile quite different ideas utilizing spatial extra dimensions were proposed and developed in recent years $[13,14,15,16,17,18,19,20,21]$. The GUT gauge symmetry manifest in higher spacetime dimensions is broken to the SM ones by the boundary conditions on a compactified orbifold. The higher dimensional SUSY is also reduced by the compactification. In this construction the mass hierarchy between doublet and triplet Higgs fields is realized without any redundant Higgs multiplets. Furthermore the dangerous dimension five proton decay and the wrong prediction of Yukawa couplings in the first two generations are avoided by appropriate choices of matter field configuration, while the gauge coupling unification at the cutoff scale $M \sim 10^{17} \mathrm{GeV}$ is maintained [15, 20, 21].

In this letter we discuss the hadronic EDMs in the orbifold GUT models which explain 
flavor structures of the fermions and sfermions by the geometrical nature of higher dimensional spaces. For definiteness we will work in a framework of SU(5) unification proposed by Hall and Nomura [21]. In this setup the matter ten-plets of the first two generations reside in the bulk, while other matter fields are located on the $\mathrm{SU}(5)$ preserving brane. The soft SUSY breaking mass terms for bulk fields are attributed to the Scherk-Schwarz mechanism $[22,23]$ with no soft masses for brane fields, resulting in non-universal mass spectrum for left-handed squarks, up-type squarks and right-handed sleptons. In generic the CP violating phases of the off-diagonal elements in the left-handed up-type quark mass matrix do not coincide with the right-handed counterparts, and they lead to the up quark CEDM enhanced by the top quark mass. We find that the predicted up quark CEDM is marginal to the current experimental limit. In this model observable lepton flavor violating processes are also predicted due to the off-diagonal terms in the right-handed slepton mass matrix. The constraint from the up quark CEDM on the orbifold GUT model is as strong as that from $\mu \rightarrow e \gamma$.

While the up quark CEDM could gives a severe constraint on the SUSY models potentially, the CEDM is suppressed under the universal scalar mass hypothesis. The orbifold GUT models, which we discuss in this paper, are the exceptions.

The proposed deuteron EDM search, which is expected to reach to the $10^{-27} \mathrm{e} \mathrm{cm} \mathrm{level,}$ is very promising [24]. The limit on the T-odd nuclear force will be lowered by two orders of magnitude over the current limit. Thus the structures of sfermion mass matrices will be well understood even if calculation of the deuteron EDM involves theoretical uncertainties arising from nuclear dynamics.

First, we recall the CEDMs of the light quarks and their experimental upper limits constrained by hadronic EDM experiments. There are several contributions to observable hadronic EDMs. The CP violating effects stem from the CEDMs $d_{q}^{C}$ and the EDMs $d_{q}$ of the light quarks $q(=u, d, s)$, and the QCD $\theta$ parameter up to the dimension five level. Using the SU(3) chiral Lagrangian technique, the quark CEDM contributions to the EDMs of the neutron and ${ }^{199} \mathrm{Hg}$ atom are evaluated as [4]

$$
\begin{aligned}
d_{n} & =-1.6 \times e\left(d_{u}^{C}+0.81 \times d_{d}^{C}+0.16 \times d_{s}^{C}\right), \\
d_{\mathrm{Hg}} & =-8.7 \times 10^{-3} \times e\left(d_{u}^{C}-d_{d}^{C}+0.005 \times d_{s}^{C}\right),
\end{aligned}
$$

when the Peccei-Quinn (PQ) mechanism works. Here $d_{n}$ is induced by the charged meson loop processes, and $d_{\mathrm{Hg}}$ arises from the nuclear force generated by meson exchanges. The quark CEDM contributions to hadronic EDMs depend on whether the PQ symmetry works to relax the strict bound on the $\theta$ term or not [25], although the numerical difference is small. The effects induced by the usual EDMs $d_{q}$ of the light quarks to $d_{n}$ are found to be comparable to those from the quark CEDMs in several approaches [26]. Meanwhile $d_{\mathrm{Hg}}$ is almost independent of the quark EDM operators due to the Schiff screening. Since we pay the attentions to the order of magnitudes of the hadronic EDM sensitivities, in this letter we assume the PQ symmetry and discard the quark EDM contributions for simplicity. The current upper bounds on the EDMs of the neutron [27] and the ${ }^{199} \mathrm{Hg}$ atom [28] are

$$
\left|d_{n}\right|<6.3 \times 10^{-26} e \mathrm{~cm}, \quad\left|d_{\mathrm{Hg}}\right|<1.9 \times 10^{-28} e \mathrm{~cm}
$$




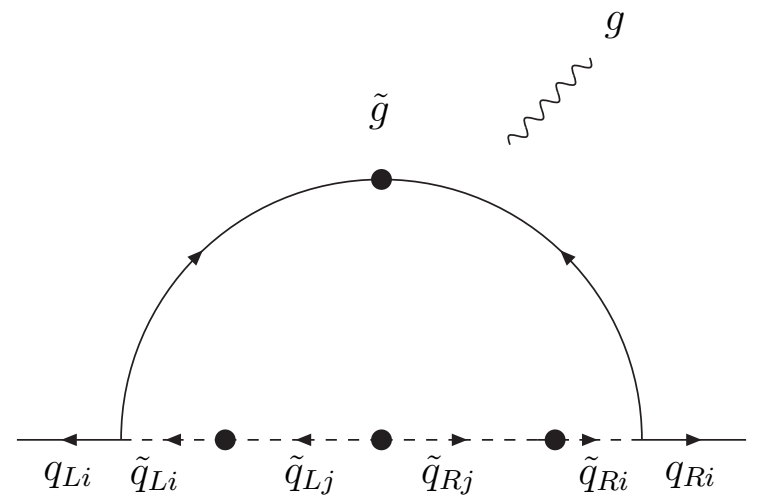

Figure 1: Diagram contributing to the CEDM of the quark when both the left-handed and right-handed squarks have $\mathrm{CP}$ violating flavor mixings. Notice that these diagrams are enhanced by the heavier quark mass due to the chirality flip in the internal line.

at $90 \%$ confidence level, respectively. In the absence of accidental cancellation among various contributions we obtain

$$
\begin{aligned}
e\left|d_{u}^{C}\right|<3.9(2.2) \times 10^{-26} e \mathrm{~cm} \\
e\left|d_{d}^{C}\right|<4.8(2.2) \times 10^{-26} e \mathrm{~cm} \\
e\left|d_{s}^{C}\right|<2.4(44) \times 10^{-25} e \mathrm{~cm}
\end{aligned}
$$

from the EDM constraints of the neutron $\left({ }^{199} \mathrm{Hg}\right.$ atom).

In discussing flavor violation in SUSY models, it is convenient to introduce the following mass insertion parameters [29]:

$$
\left(\delta_{i j}^{f}\right)_{L / R} \equiv \frac{\left(m_{\tilde{f}_{L / R}}^{2}\right)_{i j}}{\left(\bar{m}_{\tilde{f}}^{2}\right)}, \quad\left(\delta_{i}^{d}\right)_{L R} \equiv \frac{m_{d_{i}}\left(A_{i}^{(d)}-\mu \tan \beta\right)}{\bar{m}_{\tilde{d}}^{2}}, \quad\left(\delta_{i}^{u}\right)_{L R} \equiv \frac{m_{u_{i}}\left(A_{i}^{(u)}-\mu \cot \beta\right)}{\bar{m}_{\tilde{u}}^{2}},
$$

where $i, j=1,2,3$ denote the flavor indeces. Here these quantities are defined in the socalled super-CKM basis, where the corresponding quark mass matrices are diagonal and flavor violating effects are encoded into squark mass matrices.

When both the left-handed and right-handed squarks have off-diagonal components in their mass matrices, the CEDMs of the light quarks are significantly enhanced by the heavier quark mass. From Figure 1 we can estimate the quark CEDM originated from the squark flavor mixings as

$$
d_{q_{i}}^{C} \sim \frac{\alpha_{s}}{4 \pi} \frac{m_{\tilde{g}}}{\bar{m}_{\tilde{q}}^{2}} \operatorname{Im}\left[\left(\delta_{i j}^{q}\right)_{L}\left(\delta_{j}^{q}\right)_{L R}\left(\delta_{j i}^{q}\right)_{R}\right]
$$

where $\alpha_{s}=g_{s}^{2} /(4 \pi)$, and $m_{\tilde{g}}$ and $\bar{m}_{\tilde{q}}$ are the gluino and averaged squark masses. In Table 1 , we show the constraints on the mass insertion parameters from the CEDM bounds. Here we use the formulae in Ref. [10], and SUSY parameters are set to be $m_{\mathrm{SUSY}}=500 \mathrm{GeV}$ and $\tan \beta=10$. 
Table 1: Constraints on the mass insertion parameters of squarks from the EDMs of the neutron and the

${ }^{199} \mathrm{Hg}$ atom. Here, we evaluate the gluino diagram contribution to them and require them to be smaller than the experimental bounds. The superparticle masses are set to be $m_{\mathrm{SUSY}}$. The bounds on the combination of the mass insertion parameters in this table are proportional to $m_{\mathrm{SUSY}}^{2}$. Here we consider only diagrams proportional to $\tan \beta$ for the CEDMs of the strange and down quarks.

\begin{tabular}{|l|l||l|l|}
\hline \multicolumn{4}{|l|}{${ }^{199} \mathrm{Hg}$ EDM (neutron EDM $)\left(m_{\mathrm{SUSY}}=500 \mathrm{GeV}\right.$ and $\left.\tan \beta=10\right)$} \\
\hline $\operatorname{Im}\left[\left(\delta_{12}^{u}\right)_{R}\left(\delta_{21}^{u}\right)_{L}\right]$ & $0.8(1) \times 10^{-3}$ & $\operatorname{Im}\left[\left(\delta_{13}^{u}\right)_{R}\left(\delta_{31}^{u}\right)_{L}\right]$ & $3(5) \times 10^{-6}$ \\
\hline $\operatorname{Im}\left[\left(\delta_{12}^{d}\right)_{R}\left(\delta_{21}^{d}\right)_{L}\right]$ & $0.6(1) \times 10^{-3}$ & $\operatorname{Im}\left[\left(\delta_{13}^{d}\right)_{R}\left(\delta_{31}^{d}\right)_{L}\right]$ & $2(4) \times 10^{-5}$ \\
\hline $\operatorname{Im}\left[\left(\delta_{23}^{d}\right)_{R}\left(\delta_{32}^{d}\right)_{L}\right]$ & $3(0.2) \times 10^{-3}$ & & \\
\hline
\end{tabular}

The bound on $\left(\delta_{13}^{u}\right)_{L, R}$ in the up-type squark mass matrices comes from the up quark CEDM, and it severely constrains SUSY models because of the enhancement by the large top quark mass. However, the up quark CEDM is suppressed below the experimental bound when the mediation of the SUSY breaking to our visible sector is flavor-blind. The flavor violating mass terms for the squarks are radiatively generated by the Yukawa couplings. On the other hand, if the flavor universality is violated at tree level as in the orbifold GUT models, the up quark CEDM gives a stringent constraint on the models, as will be shown.

Let us briefly review the realization of realistic flavor structures of fermions and sfermions in the context of orbifold GUT models where both the GUT symmetry and SUSY are broken by the boundary conditions in extra dimensions. Especially we consider one construction of a SUSY SU(5) GUT in five dimensions proposed by Hall and Nomura [21]. The five-dimensional spacetime is postulated to be factorized into the usual four-dimensional Minkowski spacetime and the extra spatial dimension compactified on an $S^{1} / Z_{2}$ orbifold. The compactification is realized by identifying the fifth coordinate $y$ under the reflection $\mathcal{Z}: y \rightarrow-y$ and the translation $\mathcal{T}: y \rightarrow y+2 \pi R$. The physical space is a line interval, $0 \leq y \leq \pi R$, which has two branes at the orbifold fixed points $y=0$ and $y=\pi R$. The reduction of five-dimensional SUSY into four-dimensional $\mathcal{N}=1 \mathrm{SUSY}$ is attributed to the $y$-reflection $\mathcal{Z}$, and the $\mathrm{SU}(5)$ breaking comes from the translation $\mathcal{T}$ with the nontrivial action of $P=\operatorname{diag}(+,+,+,-,-)$ on a fundamental multiplet. We will see that the appropriate choice of the boundary conditions leads to the $\mathcal{N}=1$ SUSY SM.

The five-dimensional SU(5) vector multiplet lives in the bulk, and consists of a fivedimensional vector boson $A_{M}$, gauginos $\lambda$ and $\lambda^{\prime}$, and a real scalar $\sigma$. In a four-dimensional $\mathcal{N}=1$ SUSY viewpoint, the vector multiplet is rewritten by a four-dimensional vector superfield $V=\left(A_{\mu}, \lambda\right)$ and an adjoint chiral superfield $\Sigma=\left(\left(\sigma+i A_{5}\right) / \sqrt{2}, \lambda^{\prime}\right)$. The boundary conditions on the gauge multiplet are

$$
\left(\begin{array}{c}
V^{(p)} \\
\Sigma^{(p)}
\end{array}\right)\left(x^{\mu}, y\right)=\left(\begin{array}{c}
V^{(p)} \\
-\Sigma^{(p)}
\end{array}\right)\left(x^{\mu},-y\right)=p\left(\begin{array}{c}
V^{(p)} \\
\Sigma^{(p)}
\end{array}\right)\left(x^{\mu}, y+2 \pi R\right),
$$

where $p$ stands for eigenvalues of the matrix $P$. Since $p=1$ for the $\mathrm{SU}(3), \mathrm{SU}(2)$, and $\mathrm{U}(1)$ components and $p=-1$ for the others, the $\mathrm{SU}(5)$ symmetry is preserved in the bulk 
and on the brane at $y=0$, while only the SM gauge symmetry survives on the brane at $y=\pi R$. In the four-dimensional viewpoint, there appear infinite Kaluza-Klein (KK) towers of the partner states with identical quantum charges. Due to our choice of the boundary conditions, only the vector superfields with $p=1$ have zero modes which are correctly identified with the vector multiplets in the SUSY SM, whereas the vector superfields with $p=-1$ and the adjoint chiral superfield have no zero mode.

One five-dimensional hypermultiplet, which contains two complex scalars $\phi$ and $\phi^{c}$, and two chiral fermions $\psi$ and $\psi^{c}$, is written in terms of two $\mathcal{N}=1$ four-dimensional chiral multiplets, $\Phi=(\phi, \psi)$ and $\Phi^{c}=\left(\phi^{c}, \psi^{c}\right)$. The boundary conditions are

$$
\left(\begin{array}{c}
\Phi^{(p)} \\
\Phi^{c(p)}
\end{array}\right)\left(x^{\mu}, y\right)=\left(\begin{array}{c}
\Phi^{(p)} \\
-\Phi^{c(p)}
\end{array}\right)\left(x^{\mu},-y\right)=p \eta_{\Phi}\left(\begin{array}{c}
\Phi^{(p)} \\
\Phi^{c(p)}
\end{array}\right)\left(x^{\mu}, y+2 \pi R\right)
$$

where $\eta_{\Phi}=1$ or -1 . In the four-dimensional viewpoint, only the components with $p \eta_{\Phi}=1$ in $\Phi$ contain their zero modes while the other modes in $\Phi$ and $\Phi^{c}$ have masses of order of the compactification scale, as in the case of the vector multiplet.

The main purpose of the orbifold GUTs is to solve the notorious doublet-triplet splitting problem by invoking the boundary conditions. When the fundamental and anti-fundamental Higgs hypermultiplets, $\left(H, H^{c}\right)$ and $\left(\bar{H}, \bar{H}^{c}\right)$, have properties of $\eta_{H}=\eta_{\bar{H}}=-1$, only the doublet components of the KK towers of $H$ and $\bar{H}$ contain zero modes. With the help of $\mathrm{U}(1)_{R}$ symmetry, the the zero mode Higgs doublets are obliged to be massless. The U(1) $R$ symmetry also eliminates dimension four and five proton decays.

A successful gauge coupling unification is achieved at the cutoff scale $M$, although the $\mathrm{SU}(5)$ symmetry is explicitly broken on the SM brane. Let us verify that the large volume factor dilutes the SU(5) breaking effects, in the viewpoint of the four-dimensional effective theory. The SM gauge coupling constants are consists of the five-dimensional SU(5)-bulk gauge coupling constant $g_{5}$ and those from both brane-localized kinetic terms $\tilde{g}_{a}$, and written

$$
\frac{1}{g_{a}^{2}}=\frac{\pi R}{g_{5}^{2}}+\frac{1}{\tilde{g}_{a}^{2}}
$$

Here $a=1,2,3$ labels the $\mathrm{U}(1)_{Y}, \mathrm{SU}(2)_{L}$ and $\mathrm{SU}(3)_{C}$ gauge groups, respectively. The existence of the $\mathrm{SU}(5)$-breaking brane would prevent us from making any prediction without the knowledge of ultraviolet physics. This problem is easily avoided by the strong coupling hypothesis. Suppose that the gauge interaction is as strong at the cutoff scale as perturbative expansions break down. Requiring that all loop-expanded diagrams are equally weighted, we obtain $g_{5}^{2} \simeq 16 \pi^{3} / C M$ and $g_{5}^{2} \simeq 16 \pi^{2} / C_{a}$, where $C^{\prime} s$ represent group theoretical factors. Given large volume of the extra dimension such as $M R \sim O(100)$, we can ignore the contribusions from the $\mathrm{SU}(5)$-breaking brane and the bulk gauge coupling constant should be $1 / g_{*}^{2} \equiv \pi R / g_{5}^{2} \simeq 2$ for obtaining $g_{a} \simeq 0.7$. The largeness of the extra dimension leaves room for sizable deviation from the conventional MSSM prediction of the QCD coupling, in which threshold corrections from KK particles above the compactification scale enters in an SU(5)-violating manner. Since the leading power-law contributions to the gauge couplings are $\mathrm{SU}(5)$-symmetric, the renormalization group $(\mathrm{RG})$ flows of the differences among the gauge coupling constants are logarithmic and under control. The parameter set as 
$R^{-1} \sim 10^{15} \mathrm{GeV}$ and $M \sim 10^{17} \mathrm{GeV}$, for example, reconciles the the prediction of the orbifold GUT with the experimental value of the QCD coupling constant [21].

Supersymmetry breaking leading to soft breaking terms in the MSSM is also attributed to the boundary conditions. One of the most attractive features of such a boundary condition breaking is that a single parameter $\alpha$ describes all the soft terms. The supersymmetry breaking arises from the following twisted boundary conditions for $\mathrm{SU}(2)_{R}$ doublets:

$$
\left(\begin{array}{c}
\lambda^{(p)} \\
\lambda^{\prime(p)}
\end{array}\right)\left(x^{\mu}, y\right)=\left(\begin{array}{c}
\lambda^{(p)} \\
-\lambda^{\prime(p)}
\end{array}\right)\left(x^{\mu},-y\right)=e^{2 \pi i \alpha \sigma_{2}} p\left(\begin{array}{c}
\lambda^{(p)} \\
\lambda^{\prime(p)}
\end{array}\right)\left(x^{\mu}, y+2 \pi R\right),
$$

and

$$
\left(\begin{array}{c}
\phi^{(p)} \\
\phi^{c(p) \dagger}
\end{array}\right)\left(x^{\mu}, y\right)=\left(\begin{array}{c}
\phi^{(p)} \\
-\phi^{c(p) \dagger}
\end{array}\right)\left(x^{\mu},-y\right)=e^{2 \pi i \alpha \sigma_{2}} p \eta_{\Phi}\left(\begin{array}{c}
\phi^{(p)} \\
\phi^{c(p) \dagger}
\end{array}\right)\left(x^{\mu}, y+2 \pi R\right) .
$$

Given an appropriate choice of the value of $\widetilde{m} \equiv \alpha R^{-1}$, we can reproduce the weak-scale SUSY scenarios: $\alpha \sim 10^{-13}$ for $R^{-1} \sim 10^{15} \mathrm{GeV}$ for instance. The MSSM gaugino masses, which arise at the compactification scale, are given by

$$
M_{a}=\frac{g_{a}^{2}}{g_{*}^{2}} \widetilde{m}
$$

Notice that the usual gaugino mass unification is retained as in the case of the conventional SUSY GUTs. The resulting soft SUSY breaking terms for scalar zero modes in the orbifold GUT model is

$$
-\mathcal{L}_{\mathrm{soft}}=\widetilde{m}^{2}\left(|h|^{2}+\left|\phi_{B}\right|^{2}\right)-\widetilde{m}\left[3 f \phi_{B} \phi_{B} h+2 f \phi_{B} \phi_{b} h+f \phi_{b} \phi_{b} h+\text { h.c. }\right]
$$

where $h$ and $\phi_{B(b)}$ denote the Higgs doublets and bulk (brane) matter fields collectively, and $f$ the corresponding Yukawa coupling constants in the superpotential. We notice that the SUSY breaking is related with bulk fields and dictated by $\widetilde{m}$.

Let us consider the matter configuration and its consequences for the flavor structures of fermions and sfermions. There are three options for locating a matter field: in the bulk, on the $\mathrm{SU}(5)$ symmetric brane at $y=0$ or on the SM brane at $y=0$. Here we employ the following prescription suggested by Hall and Nomura: the first two families of ten-plets $T_{1,2}, T_{1,2}^{\prime}$ with $\eta_{T_{1,2}}=-\eta_{T_{1,2}^{\prime}}=1$ live in the bulk, while the third generation ten-plet $T_{3}$ and all the three families of the five-plet matters $F_{1,2,3}$ are located on the $\mathrm{SU}(5)$ symmetric brane. The first two generations of the up-type quark singlets $\bar{U}_{1,2}$ and the charged-lepton singlets $\bar{E}_{1,2}$ in the MSSM are identified with the zero modes of $T_{1,2}$, while those of the quark doublets $Q_{1,2}^{\prime}$ with those of $T_{1,2}^{\prime}$. In Figure 2 we schematically depict the geometric structure and the field configuration. The locations of the matter multiplets are determined by the combined results given by the phenomenological analysis below.

First note that the matter configuration in Figure 2 accounts for the hierarchical quark and lepton masses and mixings by a virtue of the volume suppression. The Yukawa interactions among the matter and Higgs multiplets are allowed only on the SU(5) brane. The 


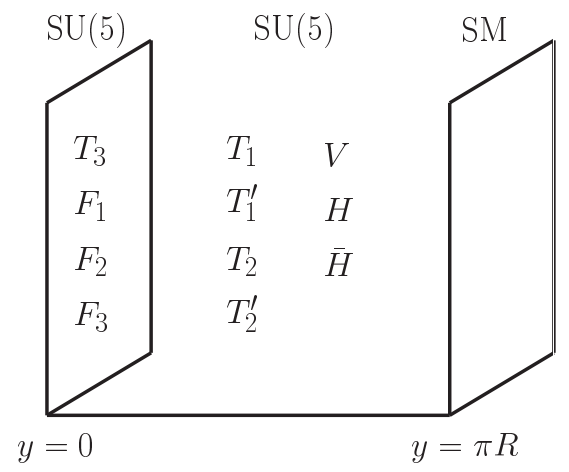

Figure 2: Geometric structure and field configuration in the fifth dimension $y$. The SU(5) vector multiplet $V$, the fundamental (anti-fundamental) Higgs hypermultiplet $H(\bar{H})$ and four ten-plet matter hypermultiplets $T_{1,2}, T_{1,2}^{\prime}$ live in the bulk. One ten-plet matter chiral superfield $T_{3}$ and three five-plet matter chiral superfields $F_{1,2,3}$ are located on the $\mathrm{SU}(5)$ symmetric brane at $y=0$. There are no localized fields on the SM brane at $y=\pi R$.

resulting Yukawa couplings in the MSSM are given by

$$
\begin{aligned}
W_{Y} \sim & \left(Q_{1}^{\prime}, Q_{2}^{\prime}, Q_{3}\right)\left(\begin{array}{cc|c}
\epsilon^{2} & \epsilon^{2} & \epsilon \\
\epsilon^{2} & \epsilon^{2} & \epsilon \\
\hline \epsilon & \epsilon & 1
\end{array}\right)\left(\begin{array}{c}
\bar{U}_{1} \\
\bar{U}_{2} \\
\bar{U}_{3}
\end{array}\right) \\
& +\left(Q_{1}^{\prime}, Q_{2}^{\prime}, Q_{3}\right)\left(\begin{array}{ccc}
\epsilon & \epsilon & \epsilon \\
\epsilon & \epsilon & \epsilon \\
\hline 1 & 1 & 1
\end{array}\right)\left(\begin{array}{c}
\bar{D}_{1} \\
\bar{D}_{2} \\
\bar{D}_{3}
\end{array}\right)+\left(\bar{E}_{1}, \bar{E}_{2}, \bar{E}_{3}\right)\left(\begin{array}{ccc}
\epsilon & \epsilon & \epsilon \\
\epsilon & \epsilon & \epsilon \\
\hline 1 & 1 & 1
\end{array}\right)\left(\begin{array}{c}
L_{1} \\
L_{2} \\
L_{3}
\end{array}\right)
\end{aligned}
$$

with coefficients of order of unity. The large extra dimension whose size is of order of $R \sim 100 M^{-1}$ suffices for the mass hierarchy, so that $\epsilon \simeq(M R)^{-1 / 2} \sim 0.1$. Only the $(3,3)$ element in the up-type Yukawa couplings and the third rows of the down-type and chargedlepton Yukawa couplings preserves SU(5) in the conventional sense, because the zero modes of the first two generations of the ten-plets stem from different bulk multiplets. Therefore the up-type quark Yukawa matrix is not symmetric and the masses of the first two generations of the down-type quarks are not associated with the counterparts of charged-leptons while retaining the bottom and tau mass unification. We should admit that the simple geometric structure of the orbifold GUT does not explain the hierarchy within the first two generations. There are several ways to avoid this difficulty. One of the options is to impose an additional $\mathrm{U}(1)$ flavor symmetry with the charge assignments such as $T_{1}^{(\prime)}(1), T_{2,3}^{(\prime)}(0), F_{1,2,3}(1)$. Given the $\mathrm{U}(1)$ flavor symmetry breaking parameter of order of $\epsilon$, more realistic Yukawa coupling matrices are obtained as

$$
f_{u} \sim\left(\begin{array}{cc|c}
\epsilon^{4} & \epsilon^{3} & \epsilon^{2} \\
\epsilon^{3} & \epsilon^{2} & \epsilon \\
\hline \epsilon^{2} & \epsilon & 1
\end{array}\right), \quad f_{d} \sim\left(\begin{array}{ccc}
\epsilon^{3} & \epsilon^{3} & \epsilon^{3} \\
\epsilon^{2} & \epsilon^{2} & \epsilon^{2} \\
\hline \epsilon & \epsilon & \epsilon
\end{array}\right), \quad f_{e} \sim\left(\begin{array}{ccc}
\epsilon^{3} & \epsilon^{3} & \epsilon^{3} \\
\epsilon^{2} & \epsilon^{2} & \epsilon^{2} \\
\hline \epsilon & \epsilon & \epsilon
\end{array}\right),
$$

instead of Eq. (13). The mass hierarchy between the top and bottom quarks is also explained 
for a small $\tan \beta$ region. In the rest of this letter, we postulate that some mechanism works to reproduce the realistic type of the Yukawa structure of Eq. (14). By introducing three generations of right-handed neutrino fields, the smallness of the neutrino masses are explained via the seesaw mechanism [30]. We can place the right-handed neutrinos either in the bulk or in the SU(5) symmetric brane. In both cases, the Dirac Yukawa couplings and the Majorana mass terms for the right-handed neutrinos are given in the SU(5) brane. Since three generations of the left-handed leptons $L_{1,2,3}$ have same geometric profiles, the large mixing angles in the neutrino sector are naturally realized.

The gauge coupling unification in the orbifold GUTs requires a lower compactification scale than the usual GUT scale in the conventional SUSY GUT scenario. Thus we might expect that dimension six nucleon decays mediated by the heavy $X$ gauge bosons with mass of order of $R^{-1}$ are dangerous. Here we recall that $\bar{U}_{1,2}, \bar{E}_{1,2}$ and $Q_{1,2}^{\prime}$ in the MSSM originate from different five-dimensional ten-plets and that the $X$ gauge bosons cannot convert $Q_{1,2}^{\prime}$ into $\bar{U}_{1,2}$ nor $\bar{E}_{1,2}$. Therefore the proton decay rates are suppressed to an acceptable level by small flavor mixings between the first two and third generations.

The same matter configuration considerably ameliorates the SUSY flavor problem. The boundary condition SUSY breaking ensures universal soft scalar masses for bulk fields with vanishing soft scalar masses for brane fields, as we mentioned above. The geometry provides the following soft terms for the zero modes:

$$
\begin{aligned}
-\mathcal{L}_{\mathrm{soft}}= & \widetilde{m}^{2}\left[\tilde{q}^{*} P_{T} \tilde{q}+\tilde{u}^{c^{*}} P_{T} \tilde{u}^{c}+\tilde{e}^{c^{*}} P_{T} \tilde{e}^{c}\right] \\
& -\widetilde{m}\left[\tilde{q}\left(f_{u}+P_{T} f_{u}+f_{u} P_{T}\right) \tilde{u}^{c} h_{u}+\tilde{q}\left(f_{d}+P_{T} f_{d}\right) \tilde{d}^{c} h_{d}\right. \\
& \left.\quad+\tilde{e}^{c}\left(f_{e}+P_{T} f_{d}\right) \tilde{l} h_{d}+\text { h.c. }\right], \\
P_{T}= & \left(\begin{array}{ccc}
1 & 0 & 0 \\
0 & 1 & 0 \\
0 & 0 & 0
\end{array}\right)
\end{aligned}
$$

at the compactification scale. At the electroweak scale we obtain universal masses for lefthanded sleptons and right-handed down-type squarks, while the third generations of lefthanded quark doublets, right-handed up-type quarks and charged sleptons are different from their first two generations in mass. The mass degeneracy especially within the first two generations adequately suppresses the most dangerous flavor violating processes caused by superparticles, such as the $K$ meson mixing and the $\mu \rightarrow e \gamma$ decay. Sizable flavor violating effects are originated only from the separation of the locations of the ten-plets. In other words it is worth investigating implications from flavor mixings between the first two generations and the third generation in left-handed squark, right-handed up-type squark and right-handed slepton sectors.

Let us explore experimental signatures from the remarkable flavor structure realized in the orbifold GUT models. The different location of the third generation ten-plet $T_{3}$ in the extra dimension from those of the first two generations induces the sfermion flavor mixing for left-handed squarks, right-handed up-type squarks and charged sleptons. Such a situation is reminiscent of the conventional SUSY SU(5) GUTs. In the SUSY SU(5) GUTs with the minimal supergravity boundary condition the renormalization group (RG) effects above the GUT scale generate similar flavor-violating terms for the sfermions via the top and bottom 
Yukawa couplings [31]. We focus on the CEDM of the up quark originated from the uptype squark mixings and LFV from the right-handed slepton sector. We will show that the observations of hadronic EDMs and the LFV $\mu \rightarrow e \gamma$ decay have a great potential to distinguish the two frameworks.

We can estimate the magnitude of the up quark CEDM using the mass insertion approximation. The Yukawa matrices are related to those in the mass eigenbasis by the following biunitary transformation: $f_{u}=V_{u_{L}}^{T} \hat{f}_{u} V_{u_{R}}, f_{d}=V_{d_{L}}^{T} \hat{f}_{d} V_{d_{R}}, f_{e}=V_{e_{R}}^{T} \hat{f}_{e} V_{e_{L}}$. Then the soft terms in the super-CKM basis are

$$
\begin{aligned}
-\mathcal{L}_{\mathrm{soft}}= & \widetilde{m}^{2}\left[\tilde{q}^{*} C_{u_{L}} \tilde{q}+\tilde{u}^{c^{*}} C_{u_{R}} \tilde{u}^{c}+\tilde{e}^{c^{*}} C_{e_{R}} \tilde{e}^{c}\right. \\
- & \widetilde{m}\left[\tilde{q}\left(\hat{f}_{u}+C_{u_{L}}^{*} \hat{f}_{u}+\hat{f}_{u} C_{u_{R}}\right) \tilde{u}^{c} h_{u}+\tilde{q}\left(V^{*} \hat{f}_{d}+C_{u_{L}}^{*} V^{*} \hat{f}_{d}\right) \tilde{d}^{c} h_{d}\right. \\
& \left.+\tilde{e^{c}}\left(\hat{f}_{e}+C_{e_{R}}^{*} \hat{f}_{e}\right) \tilde{l} h_{d}+\text { h.c. }\right],
\end{aligned}
$$

where $V=V_{u_{L}} V_{d_{L}}^{*}$ is the KM matrix. Additional flavor violating effects are controlled by

$$
\left(C_{f}\right)_{i j}=\left(V_{f} P_{T} V_{f}^{\dagger}\right)_{i j}=\mathbf{1}_{i j}-\left(V_{f}\right)_{i 3}\left(V_{f}^{*}\right)_{j 3}, \quad f=u_{L}, u_{R}, e_{R} .
$$

In generic CP violating off-diagonal elements in the left-handed up-type squark mass matrix are not related to the right-handed counterparts. Therefore we expect too large CP violation as $\operatorname{Im}\left[\left(\delta_{13}^{u}\right)_{L}\left(\delta_{31}^{u}\right)_{R}\right] \sim \epsilon^{4} \sim 10^{-4}$. Although the squark mixings are diluted by the gluino focusing effect, the CEDM of the up quark is still dangerous like the $\mu \rightarrow e \gamma$ decay.

We have performed numerical calculations clarifying the observable hadronic EDMs are indeed induced by the CP-violating sfermion mixings as well as LFV processes. Since there is a sizable mass splitting among the sfermions, we work in the basis where the sfermion mass matrices are fully diagonalized, instead of the mass insertion approximation. The CEDM of the up quark is given by

$$
d_{u}^{C}=c \frac{\alpha_{s}}{4 \pi} \frac{1}{M_{3}} \sum_{I=1}^{6}\left\{\left(-\frac{1}{3} G_{1}\left(x_{I}\right)-3 G_{2}\left(x_{I}\right)\right) \operatorname{Im}\left[\left(U_{u}^{\dagger}\right)_{1 I}\left(U_{u}\right)_{I 4}\right]\right\}
$$

where $\left(U_{u}\right)_{I i}$ relates the up-type squarks $\tilde{u}_{I}^{(m)}$ with mass $m_{I}$ to those in a flavor basis $\left(\tilde{u}_{L}^{(f)}, \tilde{u}_{R}^{(f)}\right)_{i}$ by the following unitary transformation: $\tilde{u}_{I}^{(m)}=\left(U_{u}\right)_{I i}\left(\tilde{u}_{L}^{(f)}, \tilde{u}_{R}^{(f)}\right)_{i}$. Here $c \sim 0.9$ is the QCD correction factor at low energy, $x_{I}\left(\equiv m_{I}^{2} / M_{3}^{2}\right)$ parametrizes mass ratios of the up-type squarks to the gluino, and the loop functions $G_{1}(x)$ and $G_{2}(x)$ are defined by

$$
G_{1}(x) \equiv \frac{1-x^{2}+2 x \ln x}{2(x-1)^{3}}, \quad G_{2}(x) \equiv \frac{1-4 x+3 x^{2}-2 x^{2} \ln x}{2(x-1)^{3}},
$$

where $G_{1}(1)=-1 / 6, G_{2}(1)=-1 / 3$.

In Figure 3 we show the predicted values of the CEDM of the up quark in (a) and the branching ratio for the LFV $\mu \rightarrow e \gamma$ decay in (b) as functions of $\widetilde{m}$ for $\tan \beta=3,10$. Here we set the magnitudes of sfermion mixing parameters to $\left|\left(C_{f}\right)_{13}\right|=0.01$ and $\left|\left(C_{f}\right)_{23}\right|=0.04$ at the compactification scale $R^{-1}=1 \times 10^{14} \mathrm{GeV}$, and postulate maximal CP violation. These values are naturally inferred from the corresponding CKM matrix elements, because the rotation of the ten-plets in the generation space simultaneously dictates the hierarchical 


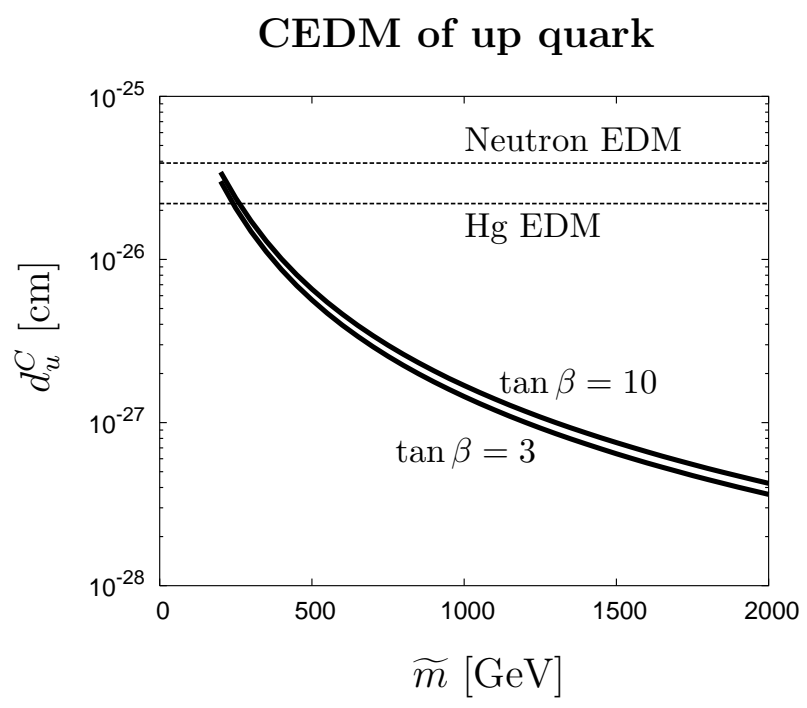

(a)

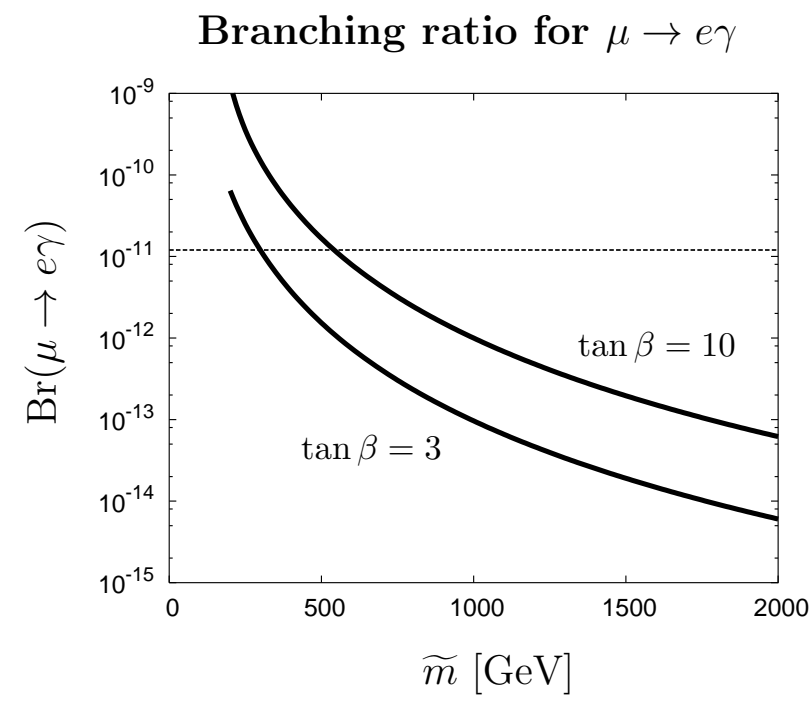

(b)

Figure 3: CEDM of up quark in (a) and branching ratio for $\mu \rightarrow e \gamma$ decay in (b) as functions of the SUSY breaking parameter $\widetilde{m}$. We set the magnitudes of the flavor violating parameters to be $\left|\left(C_{f}\right)_{13}\right|=$ $0.01,\left|\left(C_{f}\right)_{23}\right|=0.04$, and the five-dimensional gauge coupling constant to be $1 / g_{*}^{2}=1.9$ at $R^{-1}=1 \times$ $10^{14} \mathrm{GeV}$, and postulate maximal CP violation. For SUSY parameters we take $\tan \beta=3,10$ and $\mu<0$. The present experimental upper bounds are $d_{u}^{C}<2.2 \times 10^{-26} \mathrm{~cm}(\mathrm{Hg} \mathrm{EDM}), d_{u}^{C}<3.9 \times 10^{-26} \mathrm{~cm}$ (Neutron $\mathrm{EDM})$, and $\operatorname{Br}(\mu \rightarrow e \gamma)<1.2 \times 10^{-11}$.

structure of the CKM matrix and the additional sfermion mixings. The five-dimensional gauge coupling constant is taken to be $1 / g_{*}^{2}=1.9$, and the RG effects from the compactification scale to the $Z$-boson mass scale are included. The Higgs mixing parameter $\mu$ is chosen to be negative, which is preferred by considering the precise bottom-tau unification [21]. The dependence of the signature of $\mu$ on the predicted values is weak. The present experimental bounds are $d_{u}^{C}<2.2 \times 10^{-26} \mathrm{~cm}(\mathrm{Hg} \mathrm{EDM}), d_{u}^{C}<3.9 \times 10^{-26} \mathrm{~cm}$ (Neutron $\mathrm{EDM})$, and $\operatorname{Br}(\mu \rightarrow e \gamma)<1.2 \times 10^{-11}$ [32]. Due to the gluino focusing effect the mass insertion parameters in the up-type squarks at the superparticle mass scale are diminished as

$$
\left.\left.\left(\delta_{i j}^{u}\right)_{L / R}\right|_{m_{\mathrm{SUSY}}} \sim \frac{\left(\delta_{i j}^{u}\right)_{L / R}}{1+\eta\left(g_{3}^{4} / g_{*}^{4}\right)}\right|_{R^{-1}}, \quad \eta \sim 5,
$$

and their dependence on $R$ is only logarithmic, while those in the slepton sector are insensitive to RG effects. Notice that in our setup various observables, such as flavor violating processes and mass spectra, are uniquely determined by a single parameter $\widetilde{m}$, once the flavor mixing parameters are fixed. These figures demonstrate that the orbifold GUT scenario marginally survives the bounds from the hadronic EDMs and the $\mu \rightarrow e \gamma$ decay.

A new deuteron EDM experiment is proposed aiming for the sensitivity of $d_{D} \sim 10^{-27} \mathrm{e}$ $\mathrm{cm}$ [24], which is converted to the CEDM bounds as $d_{u}^{C} \sim 10^{-28} \mathrm{~cm}, d_{d}^{C} \sim 10^{-28} \mathrm{~cm}$ and 
$d_{s}^{C} \sim 10^{-26} \mathrm{~cm}$. Furthermore the MEG experiment will reach to the level of $\operatorname{Br}(\mu \rightarrow e \gamma) \sim$ $10^{-14}$ in the near future [33]. These experiments will cover the interesting parameter region in the orbifold GUT models. Null signature of the deuteron EDM or the $\mu \rightarrow e \gamma$ decay would oblige us to change the matter configuration in the extra dimension.

Let us discuss other possible choices of matter configurations in the framework of the orbifold GUTs, where SUSY is broken by the boundary conditions. We can place $T_{3}$ and $F_{1,2,3}$ on the SM brane instead of the SU(5) symmetric one. The gross flavor structures of fermions and sfermions resemble those in the Hall-Nomura model, and thus similar flavor violating effects are derived. Future improvements of the experiments may force us to eliminate the off-diagonal elements in the sfermion mass matrices if the signatures are not observed. This means that all of the ten-plets should be located on the same place in the light of the quark mixings. The first two generations of ten-plets $T_{1,2}$ are forbidden from living in the $\mathrm{SU}(5)$ brane in order to suppress the dimension six proton decays, while $T_{3}$ should be localized on one of the two branes to reproduce the large top quark mass. Thus $T_{1,2,3}$ must be located on the SM brane [16]. The locations of the three generations of fiveplets are either in the bulk or on the SM brane in order to allow the Yukawa interactions. In the bulk five-plet case, the ratio of the bottom to top Yukawa couplings is suppressed by the large volume of the extra dimension, and the soft scalar masses of the five-plets are lifted by $\widetilde{m}$. In the case where the five-plets are located on the SM brane, we obtain the same Yukawa structures as those in the MSSM. In both cases, the SU(5) relation for the Yukawa couplings is discarded, and we need some other mechanism to realize the flavor hierarchy in the fermion masses and mixings. As far as non-universal configuration for the matter multiplets is responsible for the sfermion mixings, which are dictated by the corresponding CKM matrix elements, not only LFV processes but also the hadronic EDMs is predicted to be just below the current limits. This conclusion is generic, and not altered even in extended models possessing higher-dimensional spacetimes or larger gauge groups.

The predictions of the orbifold GUTs should be compared to those of four-dimensional SUSY SU(5) GUTs. If the sfermion masses arise in a flavor-blind manner from the dynamics above the GUT scale (for example, the minimal supergravity scenario), the sfermion flavor mixings are induced only by the RG running effects from the cutoff scale $M_{G}$ and thus they are loop-suppressed. In SUSY SU(5) GUTs there exist the color triplet partners of the MSSM Higgs doublets. The Yukawa interactions mediated by the Higgs triplets with mass $M_{H^{c}}$ lead to flavor mixings in the ten-plet scalars. In the minimal SU(5) model without heavy right-handed neutrinos, the resulting flavor mixings are controlled by the CKM matrix and the top and bottom Yukawa couplings $f_{t, b}$ as

$$
\begin{aligned}
& \left(m_{\tilde{u}_{L}}^{2}\right)_{i j} \simeq-V_{i 3} V_{j 3}^{*} \frac{f_{b}^{2}}{(4 \pi)^{2}}\left(3 m_{0}^{2}+A_{0}^{2}\right)\left(2 \ln \frac{M_{G}^{2}}{M_{H_{c}}^{2}}+\ln \frac{M_{H_{c}}^{2}}{m_{\mathrm{SUSY}}^{2}}\right), \\
& \left(m_{\tilde{u}_{R}}^{2}\right)_{i j} \simeq-e^{-i \varphi_{i j}} V_{i 3}^{*} V_{j 3} \frac{2 f_{b}^{2}}{(4 \pi)^{2}}\left(3 m_{0}^{2}+A_{0}^{2}\right) \ln \frac{M_{G}^{2}}{M_{H_{c}}^{2}} \\
& \left(m_{\tilde{d}_{L}}^{2}\right)_{i j} \simeq-V_{3 i}^{*} V_{3 j} \frac{f_{t}^{2}}{(4 \pi)^{2}}\left(3 m_{0}^{2}+A_{0}^{2}\right)\left(3 \ln \frac{M_{G}^{2}}{M_{H_{c}}^{2}}+\ln \frac{M_{H_{c}}^{2}}{m_{\mathrm{SUSY}}^{2}}\right), \\
& \left(m_{\tilde{d}_{R}}^{2}\right)_{i j} \simeq 0,
\end{aligned}
$$




$$
\begin{aligned}
\left(m_{\tilde{e}_{L}}^{2}\right)_{i j} & \simeq 0 \\
\left(m_{\tilde{e}_{R}}^{2}\right)_{i j} & \simeq-V_{3 i} V_{3 j}^{*} \frac{3 f_{t}^{2}}{(4 \pi)^{2}}\left(3 m_{0}^{2}+A_{0}^{2}\right) \ln \frac{M_{G}^{2}}{M_{H_{c}}^{2}},
\end{aligned}
$$

with $i \neq j$, where $\varphi_{i j} \equiv \varphi_{i}-\varphi_{j}$ parametrizes the GUT-origin phases independent of the KM phase. Here $m_{0}$ and $A_{0}$ are the universal soft scalar mass and the trilinear coupling, respectively. Although both the left-handed and right-handed up-type squarks have the off-diagonal components with different CP phases, the induced CEDM of the up quark is suppressed by the fourth power of bottom Yukawa coupling in addition to the loop suppression [9] ${ }^{1}$. For example, we obtain $d_{u}^{C} \sim 10^{-28} \mathrm{~cm}$ for $m_{\mathrm{SUSY}}=500 \mathrm{GeV}$ and $\tan \beta \simeq 35$. Even for a large $\tan \beta$ region the predicted hadronic EDMs are well below the current upper limits. If we observe the deuteron EDM above the $10^{-27} e \mathrm{~cm}$ level, it would be attributed to the down or strange quark contribution coming from other physics in the context of four-dimensional GUTs. On the other hand, the right-handed slepton mixings involve the large top Yukawa coupling, so that LFV decay processes are within the scope of future experiments, although the various contributions tend to cancel out [35]. We emphasize that by combining the results of the hadronic EDM and LFV searches we can distinguish the orbifold framework from the conventional SU(5) SUSY GUTs.

It is also important to measure various hadronic EDM processes, because the light quark CEDM contributions to hadronic EDMs are quite different. By investigating the correlation of the observed hadronic EDMs, we might find which contribution dominates over. If we identify the origin, it would give us crucial hints for the physics beyond the SM.

In this letter we have investigated the hadronic EDM constraints on the orbifold GUT framework, which explains the fermion and sfermion flavor structures due to an appropriate choice of the locations of the matter fields in extra dimensions. For definiteness we have worked in one construction based on SU(5) proposed by Hall and Nomura. In the setup the first two generations of the ten-plet matter fields live in the bulk as the gauge and Higgs multiplets, while the third generation ten-plet and all of the three five-plet matters are localized on the SU(5) conserving brane. Given the matter geometry, (i) realistic fermion masses and mixings are reproduced retaining the bottom-tau mass unification, (ii) flavor changing processes via superparticles are suppressed due to the partial mass degeneracy, and (iii) dimension six nucleon decays mediated by the heavy gauge bosons are avoided. We explore observable flavor-violating signatures coming from the non-degenerate sfermion mass spectra for the ten-plet sector: the left-handed squarks, the up-type squarks and the singlet sleptons. We have pointed out that CP-violating up-type squark mixings between the first and third generations in both the left- and right-handed sectors induces a sizable CEDM of the up quark, which is enhanced by the large top quark mass. As a result, the hadronic EDM constraints on the orbifold GUT framework are predicted to be close to the current experimental bounds as well as that from $\mu \rightarrow e \gamma$ decay. On the contrary, in the context

\footnotetext{
${ }^{1}$ The radiative corrections to the off-diagonal terms for left- and right-handed up squarks may be generated by the top quark Yukawa coupling, when the misalignment between the Yukawa couplings in the MSSM and the GUTs is introduced, such as by the higher dimensional operators [34]. However, the up quark CEDM is still expected to be smaller than $\sim 10^{-28} \mathrm{~cm}$ since the correction is suppressed by loop factors.
} 
of conventional four-dimensional SUSY GUTs, the induced up quark CEDM is negligible whereas a detectable rate for the $\mu \rightarrow e \gamma$ decay is expected. The future EDM and LFV experiments will act as good probes to discriminate various SUSY GUT models.

\section{Acknowledgments}

The work of J.H. is supported in part by the Grant-in-Aid for Science Research, Ministry of Education, Science and Culture, Japan (No.13135207, No.15540255 and No.17043003). That of M.K. is supported in part by JSPS.

\section{References}

[1] J. Hisano and Y. Shimizu, Phys. Lett. B 565 (2003) 183.

[2] M. Ciuchini, A. Masiero, L. Silvestrini, S. K. Vempati and O. Vives, Phys. Rev. Lett. 92 (2004) 071801.

[3] J. Hisano and Y. Shimizu, Phys. Lett. B 581 (2004) 224.

[4] J. Hisano and Y. Shimizu, Phys. Rev. D 70 (2004) 093001.

[5] K. Abe et al. [BELLE Collaboration], arXiv:hep-ex/0409049; B. Aubert et al. [BABAR Collaboration], arXiv:hep-ex/0408072.

[6] T. Moroi, Phys. Lett. B 493 (2000) 366.

[7] S. Dimopoulos and L. J. Hall, Phys. Lett. B 344 (1995) 185.

[8] I. B. Khriplovich and K. N. Zyablyuk, Phys. Lett. B 383 (1996) 429.

[9] A. Romanino and A. Strumia, Nucl. Phys. B 490 (1997) 3.

[10] J. Hisano, M. Kakizaki, M. Nagai and Y. Shimizu, Phys. Lett. B 604 (2004) 216.

[11] N. Sakai and T. Yanagida, Nucl. Phys. B 197 (1982) 533; S. Weinberg, Phys. Rev. D 26 (1982) 287.

[12] For examples of the four-dimensional SUSY GUTs, see following articles, J. Hisano, T. Moroi, K. Tobe and T. Yanagida, Phys. Lett. B 342 (1995) 138; K. S. Babu and S. M. Barr, Phys. Rev. D 48 (1993) 5354; Phys. Rev. D 65 (2002) 095009; N. Maekawa, Prog. Theor. Phys. 107 (2002) 597; N. Maekawa and T. Yamashita, Prog. Theor. Phys. 107 (2002) 1201; T. Yanagida, Phys. Lett. B 344 (1995) 211; T. Hotta, K. I. Izawa and T. Yanagida, Phys. Rev. D 53 (1996) 3913; Phys. Rev. D 54 (1996) 6970.

[13] Y. Kawamura, Prog. Theor. Phys. 105 (2001) 999.

[14] G. Altarelli and F. Feruglio, Phys. Lett. B 511 (2001) 257.

[15] L. J. Hall and Y. Nomura, Phys. Rev. D 64 (2001) 055003.

[16] A. Hebecker and J. March-Russell, Nucl. Phys. B 613 (2001) 3.

[17] A. Hebecker and J. March-Russell, Nucl. Phys. B 625 (2002) 128. 
[18] T. Asaka, W. Buchmuller and L. Covi, Phys. Lett. B 523 (2001) 199.

[19] L. Hall, J. March-Russell, T. Okui and D. R. Smith, JHEP 0409 (2004) 026.

[20] L. J. Hall and Y. Nomura, Phys. Rev. D 65 (2002) 125012.

[21] L. J. Hall and Y. Nomura, Phys. Rev. D 66 (2002) 075004.

[22] J. Scherk and J. H. Schwarz, Phys. Lett. B 82 (1979) 60; J. Scherk and J. H. Schwarz, Nucl. Phys. B 153 (1979) 61.

[23] R. Barbieri, L. J. Hall and Y. Nomura, Phys. Rev. D 66 (2002) 045025.

[24] Y. K. Semertzidis et al. [EDM Collaboration], AIP Conf. Proc. 698 (2004) 200.

[25] I. I. Y. Bigi and N. G. Uraltsev, Sov. Phys. JETP 73 (1991) 198.

[26] M. Pospelov and A. Ritz, arXiv:hep-ph/0504231, and references therein.

[27] P. G. Harris et al., Phys. Rev. Lett. 82 (1999) 904.

[28] M. V. Romalis, W. C. Griffith and E. N. Fortson, Phys. Rev. Lett. 86 (2001) 2505.

[29] L. J. Hall, V. A. Kostelecky and S. Raby, Nucl. Phys. B 267 (1986) 415; F. Gabbiani, E. Gabrielli, A. Masiero and L. Silvestrini, Nucl. Phys. B 477 (1996) 321.

[30] M. Gell-Mann, P. Ramond and R. Slansky, Proceedings of the Supergravity Stony Brook Workshop, New York, 1979, eds. P. Van Nieuwenhuizen and D. Freedman (NorthHolland, Amsterdam); T. Yanagida, Proceedings of the Workshop on Unified Theories and Baryon Number in the Universe, Tsukuba, Japan 1979 (edited by A. Sawada and A. Sugamoto, KEK Report No. 79-18, Tsukuba).

[31] R. Barbieri and L. J. Hall, Phys. Lett. B 338 (1994) 212; R. Barbieri, L. J. Hall and A. Strumia, Nucl. Phys. B 445 (1995) 219; R. Barbieri, L. J. Hall and A. Strumia, Nucl. Phys. B 449 (1995) 437.

[32] M. L. Brooks et al. [MEGA Collaboration], Phys. Rev. Lett. 83 (1999) 1521.

[33] T. Mori, Talk given at the 12th International Conference on Supersymmetry and Unification of Fundamental Interactions, SUSY 2004, 17-23 June 2004, Tsukuba, Japan.

[34] N. Arkani-Hamed, H. C. Cheng and L. J. Hall, Phys. Rev. D 53 (1996) 413; J. Hisano, D. Nomura, Y. Okada, Y. Shimizu and M. Tanaka, Phys. Rev. D 58 (1998) 116010.

[35] J. Hisano, T. Moroi, K. Tobe and M. Yamaguchi, Phys. Lett. B 391 (1997) 341 [Erratum-ibid. B 397 (1997) 357]. 